\title{
Hierarchical ZnO Nanowire-Nanosheet Architectures for High Power Conversion Efficiency in Dye-Sensitized Solar Cells
}

\author{
Feng Xu, ${ }^{*,+,}$ Min Dai, ${ }^{\ddagger}$ Yinong Lu, ${ }^{\ddagger}$ and Litao Sun*, \\ Key Laboratory of MEMS of Ministry of Education, Southeast University, Nanjing 210096, People's Republic \\ of China, and State Key Laboratory of Materials-Oriented Chemical Engineering, Nanjing University of \\ Technology, Nanjing 210009, People's Republic of China
}

Received: October 30, 2009; Revised Manuscript Received: December 15, 2009

\begin{abstract}
We present a two-step synthesis process to produce hierarchical $\mathrm{ZnO}$ nanoarchitectures that involves the preparation of $\mathrm{ZnO}$ nanosheet arrays by the pyrolysis of the precursor $\mathrm{Zn}_{5}(\mathrm{OH})_{8} \mathrm{Cl}_{2}$ electrodeposited on conductive glass substrates, followed by the aqueous chemical growth (ACG) of dense $\mathrm{ZnO}$ single-crystalline nanowires on the surfaces of the primary $\mathrm{ZnO}$ nanosheets. The dye-sensitized solar cell (DSSC) based on the hierarchical $\mathrm{ZnO}$ nanowire-nanosheet architectures showed a power conversion efficiency of $4.8 \%$, which is nearly twice as high as that of the DSSC constructed using a photoanode of bare $\mathrm{ZnO}$ nanosheet arrays. The better photovoltaic performance of hierarchical $\mathrm{ZnO}$ nanoarchitecture DSSC was due to a better dye loading and light harvesting as a consequence of the enlargement of the internal surface area within the photoanode. Moreover, the improved performance for the DSSC with the hierarchical $\mathrm{ZnO}$ nanowire-nanosheet architectures may be also ascribed to more light scattering behavior through extending the optical path length within the photoanode so as to increase the light harvesting. The results demonstrate potential application of the hierarchical $\mathrm{ZnO}$ nanoarchitectures derived from $\mathrm{ZnO}$ nanosheet arrays for highly efficient DSSCs.
\end{abstract}

\section{Introduction}

Zinc oxide ( $\mathrm{ZnO})$, an important II-VI semiconductor with a wide bandgap of $3.37 \mathrm{eV}$ and a large exciton binding energy of $60 \mathrm{meV},{ }^{1,2}$ has attracted extensive attention as a promising candidate for applications in photocatalysis, ${ }^{3}$ light emitting diodes (LED) ${ }^{4}$ nanogenerators, ${ }^{5}$ dye-sensitized solar cells (DSSCs) ${ }^{6-8}$ etc. As one special kind of solar cells, DSSCs possess advantages of low cost and ease of fabrication compared to traditional silicon solar cells. In fact, the DSSCs with a power conversion efficiency of over $10 \%$ have been achieved using a photoanode of $\mathrm{TiO}_{2}$ nanocrystallite films sensitized by rutheniumbased dyes. ${ }^{9,10}$ At present, an undeniable fact is that the efficiency of DSSCs based on $\mathrm{ZnO}$ is still low as compared to $\mathrm{TiO}_{2}$-based DSSCs. The low efficiency in $\mathrm{ZnO}$-based cells could likely be ascribed to the accumulation of $\mathrm{Zn}^{2+} /$ dye complexes instead of the effective chemical bonding between $\mathrm{ZnO}$ and dye molecules as well as lower electron injection efficiency and lower dye regeneration efficiency. ${ }^{11}$ Nevertheless, considerable interest still focuses on $\mathrm{ZnO}$-based solar cells due to significantly high electron mobility and more flexibility in synthesis and morphologies in comparison with $\mathrm{TiO}_{2}$. Better electron transport can in principle result in more efficient electron collection, provided that other adverse problems (instability in acidic electrolytes, injection efficiency, and recombination loss) can be solved.

So far, various $\mathrm{ZnO}$ nanostructures have been extensively investigated for DSSCs. In the early reports on $\mathrm{ZnO}$-based DSSCs, ZnO nanoparticles were often used as the photoanode prepared by a conventional doctor blade technique. ${ }^{12,13}$ Electrons are photoexcited within the dye and are subsequently injected

* To whom correspondence should be addressed. Phone: +86-2583793437. Fax: +86-25-83792939. E-mail: fxu@seu.edu.cn.; E-mail: slt@seu.edu.cn.

Southeast University.

* Nanjing University of Technology. into the $\mathrm{ZnO}$ nanoparticles. Photogenerated electrons diffuse through the sintered nanoparticle film to the collection electrode, via a series of interparticle hopping steps. ${ }^{7}$ However, the excess electron hopping through the interparticle barriers could result in a long dwell time within individual particles and thus increase probability of charge recombination between the injected electrons and oxidized dye or redox species in the electrolyte. Recently, ZnO arrays of 1D nanostructures, such as nanowires ${ }^{6,14-16}$ and nanotubes, ${ }^{17}$ have been widely utilized to significantly enhance the electron transport velocity within the photoanode by providing a direct conduction pathway for the rapid collection of photogenerated electrons, which reduces the number of interparticle hops. But, insufficient internal surface area of these 1D nanostructure arrays limits the power conversion efficiency at a relatively low level (e.g., only $1.5 \%$ for $\mathrm{ZnO}$ nanowires ${ }^{6}$ ) owing to deficient dye loading and light harvesting. In this regard, Cheng et al. ${ }^{18}$ and Jiang et al. ${ }^{19}$ respectively fabricated the branched $1 \mathrm{D} \mathrm{ZnO}$ nanostructures consisting of upstanding nanowires and outstretched branches via a simple low-temperature hydrothermal method. The 1D secondary branches directly attached to the main $\mathrm{ZnO}$ nanowire backbone could both afford a direct conduction pathway and achieve the higher dye adsorption, motivating further improvement in the power conversion efficiency of DSSCs $(1.51 \%$ for Cheng et al. and $1.9 \%$ for Jiang et al.). Subsequently, $\mathrm{Ku}$ et al. ${ }^{20}$ also presented an approach to enlarge the internal surface area within the photoanode by introducing nanoparticles into original 1D nanowires and obtained a high efficiency ZnO-based DSSC of $3.2 \%$. In addition, $2 \mathrm{D} \mathrm{ZnO}$ nanostructures have also been studied for DSSC applications on account of the fact that they also have a large specific surface area. For instance, the DSSCs constructed using upright-standing $\mathrm{ZnO}$ nanosheet films exhibit a very high conversion efficiency of $3.9 \% .^{21}$ Other $\mathrm{ZnO}$ nanostructures, such as nanobelts ${ }^{22}$ and nanotetrapods, ${ }^{23,24}$ are also desirable candidates for use in DSSC photoanode. Note that for these 

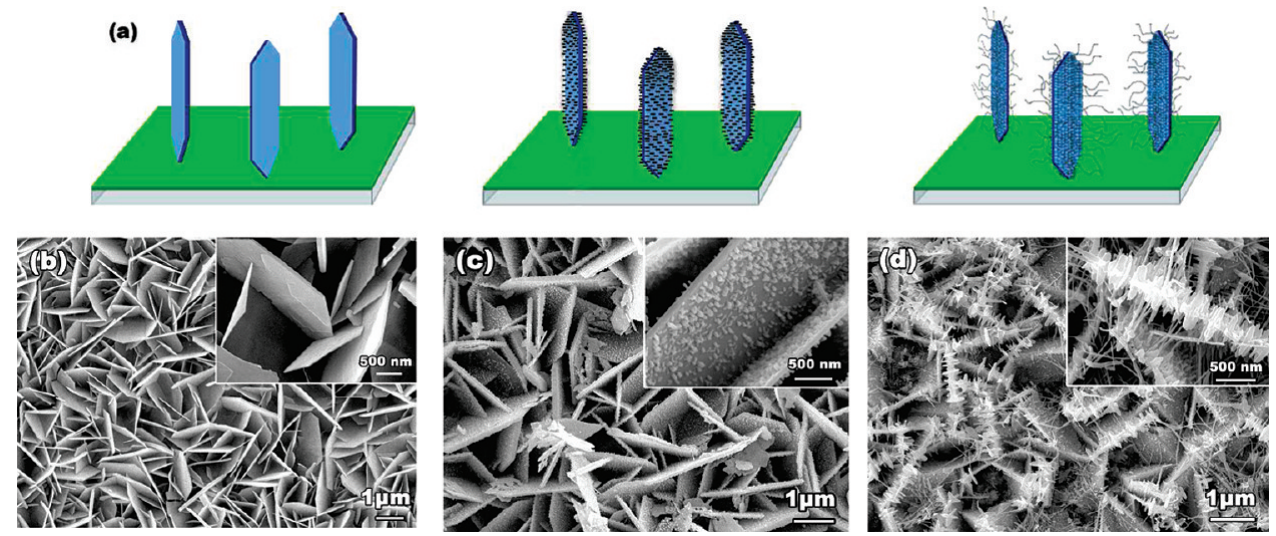

Figure 1. (a) The schematic evolution process of the hierarchical $\mathrm{ZnO}$ nanoarchitectures derived from $\mathrm{ZnO}$ nanosheet arrays and (b-d) top-view SEM images of the hierarchical $\mathrm{ZnO}$ nanowire-nanosheet architectures obtained by aqueous chemical growth after 0,1 , and $4 \mathrm{~h}$, respectively. The insets in parts $\mathrm{b}-\mathrm{d}$ correspond to their magnified images.

nanostructures the specific surface area is not the only factor that determines the photovoltaic efficiency of the DSSC. Solarcell performance is generally believed to be significantly affected by the geometrical structure of the photoanode films, which provides particular properties in terms of the electron transport and/or light propagation. Therefore, as a photoanode, besides the dye loading and light harvesting, the morphology of $\mathrm{ZnO}$ nanostructured film also plays a significant role in enhancing the electron conduction.

On the basis of the above discussions, in this paper, we shall report a DSSC with a hierarchical $\mathrm{ZnO}$ nanoarchitecture film photoanode derived from $\mathrm{ZnO}$ nanosheet arrays by a facile twostep synthesis process and compare its performance with that of the DSSC with an upright-standing $\mathrm{ZnO}$ nanosheet film as photoanode. The hierarchical $\mathrm{ZnO}$ nanoarchitectures consist of the framework of $\mathrm{ZnO}$ nanosheet arrays and dense nanowires grown on the primary $\mathrm{ZnO}$ nanosheets. This is based on the consideration that the nanosheet arrays alone may not capture the photons completely due to the existence of gaps inherent in the morphology. The hierarchical $\mathrm{ZnO}$ nanowire-nanosheet architectures, however, have nanoscale branches that stretch to fill these gaps and, therefore, provide both a larger internal surface area and a direct pathway for electron transport along the channels from the branched nanowires to the nanosheet backbone. We shall demonstrate that using the hierarchical nanowire-nanosheet architecture photoanode helps us to greatly increase the dye loading and light harvesting, while retain good electron conductivity as in the upright-standing nanosheet photoanode.

\section{Experimental Section}

The hierarchical $\mathrm{ZnO}$ nanoarchitectures derived from electrodeposited $\mathrm{ZnO}$ nanosheet arrays were synthesized by combining a low-temperature electrodeposition process and subsequent aqueous chemical growth (ACG). First, the arrays of hexagonal $\mathrm{Zn}_{5}(\mathrm{OH})_{8} \mathrm{Cl}_{2}$ nanosheet were electrodeposited on indium tin oxide (ITO, In: $\mathrm{SnO}_{2}$ ) glass substrates $(10 \Omega / \square)$ in the aqueous electrolyte of $0.05 \mathrm{M} \mathrm{Zn}\left(\mathrm{NO}_{3}\right)_{2}$ and $0.1 \mathrm{M} \mathrm{KCl}$ with a three-electrode electrochemical configuration that can be found elsewhere. ${ }^{25}$ In brief, an ITO substrate, a Pt electrode, and a saturated calomel electrode (SCE) were used as the working electrode, the counter electrode, and the reference electrode, respectively. The electrodeposition was performed at $-1.1 \mathrm{~V}$ and $50{ }^{\circ} \mathrm{C}$ for $30 \mathrm{~min}$. Upon thermal treatment at 350 ${ }^{\circ} \mathrm{C}$ or above in air, the obtained $\mathrm{Zn}_{5}(\mathrm{OH})_{8} \mathrm{Cl}_{2}$ nanosheets can be converted into $\mathrm{ZnO}$ nanosheets via solid-state crystal phase transformation, without altering the hexagonal sheetlike shape. Second, the hierarchical $\mathrm{ZnO}$ nanoarchitectures were grown by immersing the $\mathrm{ZnO}$ nanosheet substrate obtained from the first step into the bottle filled with an equimolar concentration $(0.005$ M) aqueous solution of $\mathrm{Zn}\left(\mathrm{NO}_{3}\right)_{2}$ and $\mathrm{C}_{6} \mathrm{H}_{12} \mathrm{~N}_{4}$ (HMT). ${ }^{26}$ Then, the bottle was sealed and kept at a constant temperature of 90 ${ }^{\circ} \mathrm{C}$. The hierarchical $\mathrm{ZnO}$ nanoarchitectures rooted in $\mathrm{ZnO}$ nanosheet arrays were finally rinsed with deionized water and dried in air at $60{ }^{\circ} \mathrm{C}$.

The morphology and phase identification of the products were investigated by scanning electron microscopy (SEM, JSM-5900, JEOL Ltd., Japan) and powder X-ray diffractometry (XRD, ARL

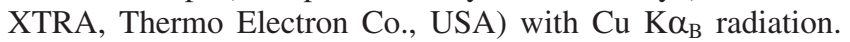
Further structural analysis of individual $\mathrm{ZnO}$ nanowires was carried out using transmission electron microscopy (TEM) and high-resolution TEM (HRTEM, JEM-2100, JEOL Ltd., Japan).

The fabrication and characterization of DSSCs were performedasfollows: Dyeing of thehierarchicalZnOnanowire-nanosheet architectures on ITO substrates were performed in a $0.3 \mathrm{mM}$ ethanolic solution containing cis-bis(isothiocyanato)bis(2,2'bipyridyl-4,4'-dicarboxylato)-ruthenium(II)bis-tetrabutylammonium (N719, Solaronix) dye at $80{ }^{\circ} \mathrm{C}$ for $2 \mathrm{~h}$. The samples were then rinsed with ethanol to remove excess dye adsorbed and dried in air at room temperature. The sensitized electrode was sandwiched together with a platinum-coated ITO counter electrode separated by about $50 \mu \mathrm{m}$ thick spacers. Sheets of weigh paper were cut into small pieces with $2 \times 6 \mathrm{~mm}$ in dimension and used as spacers. The internal space of the two electrodes was filled with a liquid electrolyte composed of 0.05 $\mathrm{M} \mathrm{I}_{2}, 0.5 \mathrm{M} \mathrm{LiI}, 0.5 \mathrm{M}$ 4-tert-butylpyridine, and $0.6 \mathrm{M}$ methylhexylimidazolium iodide in acetonitrile by capillary action. The photocurrent-photovoltage (IV) relationship of the cell was measured under a self-made AM $1.5 \mathrm{G}$ simulated sunlight $\left(100 \mathrm{~mW} / \mathrm{cm}^{2}\right)$ system with a CHI-660D electrochemical working station. The active cell area was typically $\sim 0.64$ $\mathrm{cm}^{2}$ (defined by a lightproof black mask). The amount of dye loading was determined by deadsorbing the dye within the photoanode with $0.1 \mathrm{M} \mathrm{NaOH}$ solution and measuring the optical absorbance with a Shimadzu UV-3101PC UV-vis-nearinfrared spectrophotometer.

\section{Results and Discussion}

The evolution process of the hierarchical $\mathrm{ZnO}$ nanoarchitectures derived from $\mathrm{ZnO}$ nanosheet arrays is schematically illustrated in Figure 1a and its corresponding SEM images are shown in parts $b-d$ of Figure 1. Figure $1 b$ shows that the 

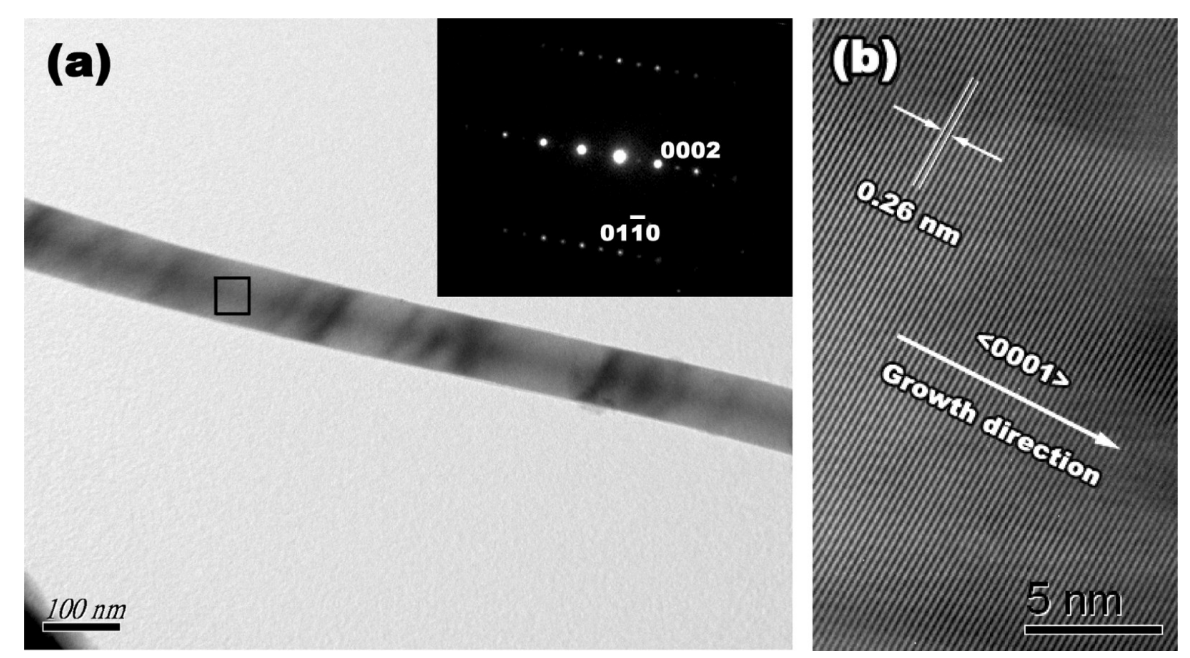

Figure 2. (a) Low-resolution TEM image of an individual $\mathrm{ZnO}$ nanowire from the hierarchical $\mathrm{ZnO}$ nanowire-nanosheet architectures as shown in Figure 1d and its corresponding SAED pattern (inserted at the up-right corner). (b) HRTEM image taken from the area marked with the black frame in (a).
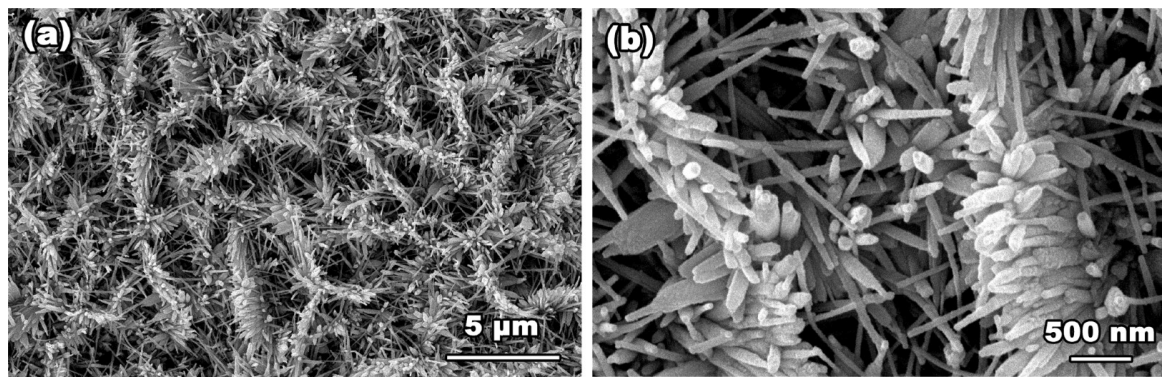

Figure 3. (a) Low- and (b) high-magnification top-view SEM images of the hierarchical $\mathrm{ZnO}$ nanowire-nanosheet architectures after aqueous chemical growth for $12 \mathrm{~h}$.

original $\mathrm{ZnO}$ nanosheet arrays were first prepared on the ITO substrate on a large scale by the pyrolysis of $\mathrm{Zn}_{5}(\mathrm{OH})_{8} \mathrm{Cl}_{2}$ nanosheets directly electrodeposited on the ITO substrate at a low temperature of $50{ }^{\circ} \mathrm{C}$. These $\mathrm{ZnO}$ nanosheets standing on the substrate exhibit the regular hexagonal end planes with 4-5 $\mu \mathrm{m}$ in diameter and about $100 \mathrm{~nm}$ in thickness. We can also see that the $\mathrm{ZnO}$ nanosheets are almost vertical to the ITO substrate, i.e., the $c$ axes of the sheetlike $\mathrm{ZnO}$ crystals are parallel to the substrate. Moreover, it can be observed from the inset in Figure $1 \mathrm{~b}$ that the surface of $\mathrm{ZnO}$ nanosheets is quite smooth, and there is a mass of gaps existing among the vertically aligned sheetlike crystals. Subsequently, the aqueous chemical growth (ACG) method similar to our previous work ${ }^{26}$ was used to grow the hierarchical $\mathrm{ZnO}$ nanoarchitectures on the ITO substrate with $\mathrm{ZnO}$ nanosheet arrays. After a 1-h growth duration, large numbers of tiny $\mathrm{ZnO}$ crystallites began to germinate uniformly on both surfaces of the primary $\mathrm{ZnO}$ nanosheets, as displayed in Figure 1c and its inset. As the growth period was increased to $4 \mathrm{~h}$, one can find that many thin and long $\mathrm{ZnO}$ nanowires rooted in the $\mathrm{ZnO}$ nanosheets have outstretched to fill the intervals between the primary $\mathrm{ZnO}$ nanosheets, as shown in Figure 1d. The magnified SEM image in the inset of Figure 1d further reveals that $\mathrm{ZnO}$ nanowires grew symmetrically on both surfaces of each $\mathrm{ZnO}$ nanosheet, which can greatly enhance the fill rate in the intervals. These $\mathrm{ZnO}$ nanowires intercrossed or interconnected one another, forming an intricate transportation network that could provide potential application for gathering and transferring the photogenerated electrons in DSSCs.

Generally, the electronic conductivity of $\mathrm{ZnO}$ nanowires is dependent on their crystallinity to some extent. Thus, the structural characterization of $\mathrm{ZnO}$ nanowires was performed by
TEM and HRTEM. To prepare TEM samples, the hierarchical $\mathrm{ZnO}$ nanowire - nanosheet architectures corresponding to Figure $1 \mathrm{~d}$ were scraped from the ITO substrate and dispersed by ethanol onto an amorphous holey carbon film coated copper grid. Thereinto, only nanowires were selected for observation. Figure 2a shows the low-resolution TEM image of an individual $\mathrm{ZnO}$ nanowire with diameter of $\sim 80 \mathrm{~nm}$. The corresponding SAED pattern (inserted at the up-right corner of Figure 2a) of the $\mathrm{ZnO}$ nanowire can be indexed to the wurtzite structure of hexagonal $\mathrm{ZnO}$ and indicates its single-crystalline nature and its growth direction along [0001]. The typical HRTEM image, taken from the area marked with the black frame in Figure 2a, is illustrated in Figure $2 b$. The crystal lattice fringes are clearly detected and the average distance between the adjacent lattice planes is 0.26 $\mathrm{nm}$ that also corresponds well to the interplanar distance of the $\{0001\}$ crystal planes of the wurtzite $\mathrm{ZnO}$. More importantly, no crystal dislocations and defects are found from the HRTEM image, showing that $\mathrm{ZnO}$ nanowires derived from $\mathrm{ZnO}$ nanosheets in the present system possess high crystalline quality.

Figure 3 presents the large-scale and magnified SEM images of the adequately grown $\mathrm{ZnO}$ nanowire-nanosheet architectures after a 12-h reaction time. Interestingly, the concrete sheetlike $\mathrm{ZnO}$ crystals as shown in Figure 1d are no longer recognized in Figure 3a, but the framework with the sheetlike morphology still exists over the whole ITO substrate. The sheetlike framework seems to consist of many vertically aligned "nanosheets"; only these "nanosheets" are composed of a great deal of nanowires resulted from the original nanosheets with concrete shapes, which shows that $\mathrm{ZnO}$ nanosheets have entirely converted to $\mathrm{ZnO}$ nanowires. Moreover, it can be seen from the magnified SEM image shown in Figure $3 b$ that the gaps 


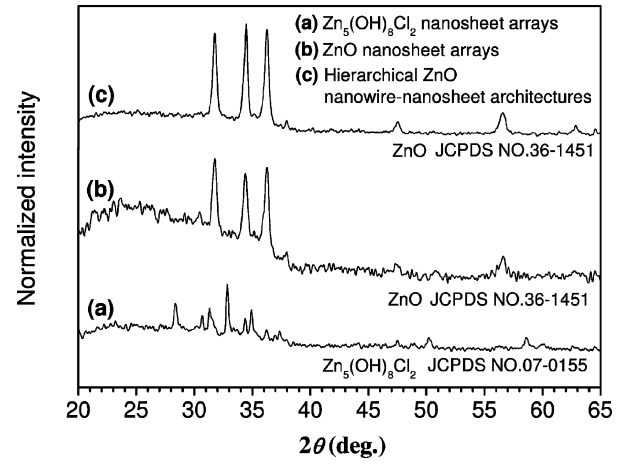

Figure 4. $\mathrm{XRD}$ patterns of (a) the as-electrodeposited $\mathrm{Zn}_{5}(\mathrm{OH})_{8} \mathrm{Cl}_{2}$ nanosheet arrays, (b) the $\mathrm{ZnO}$ nanosheet arrays prepared by pyrolysis of $\mathrm{Zn}_{5}(\mathrm{OH})_{8} \mathrm{Cl}_{2}$ nanosheet arrays, and (c) the hierarchical $\mathrm{ZnO}$ nanowire-nanosheet architectures derived from the $\mathrm{ZnO}$ nanosheet arrays by ACG method.

between adjacent sheetlike skeletons have been densely filled with the randomly oriented $\mathrm{ZnO}$ nanowires.

Structural analysis of the hierarchical $\mathrm{ZnO}$ nanowire-nanosheet architectures corresponding to Figure 3 was carried out using $\mathrm{XRD}$. Figure $4 \mathrm{c}$, the XRD pattern of the hierarchical $\mathrm{ZnO}$ nanowire-nanosheet architectures, shows that all diffraction peaks can be indexed to wurtzite (hexagonal) structured $\mathrm{ZnO}$ (space group $P 6_{3} m c$ ) with cell parameters $a=3.249 \AA$ and $c$ $=5.206 \AA$, which is in good agreement with the literature values (JCPDS card, No. 36-1451). No characteristic peaks of other impurities were detected in the pattern, suggesting that only single-phase $\mathrm{ZnO}$ sample was formed by ACG method. In addition, XRD patterns of the as-electrodeposited $\mathrm{Zn}_{5}(\mathrm{OH})_{8} \mathrm{Cl}_{2}$ nanosheet arrays and the $\mathrm{ZnO}$ nanosheet arrays prepared by pyrolysis of $\mathrm{Zn}_{5}(\mathrm{OH})_{8} \mathrm{Cl}_{2}$ nanosheet arrays are also displayed in parts a and $b$ of Figure 4, respectively. Herein, upon thermal treatment at $350{ }^{\circ} \mathrm{C}$ or above in air, the $\mathrm{Zn}_{5}(\mathrm{OH})_{8} \mathrm{Cl}_{2}$ nanosheets can be converted into $\mathrm{ZnO}$ nanosheets via solid-state transformation of crystal structure in terms of the proposed reaction eq 1 , without altering the hexagonal sheetlike shape. The solidstate transformation of crystal structure is often utilized to obtain other $\mathrm{ZnO}$ structures with novel morphology. ${ }^{27-29}$

$$
\mathrm{Zn}_{5}(\mathrm{OH})_{8} \mathrm{Cl}_{2} \rightarrow 5 \mathrm{ZnO}+3 \mathrm{H}_{2} \mathrm{O} \uparrow+2 \mathrm{HCl} \uparrow
$$

Note that the formation of $\mathrm{ZnO}$ nanosheets is pivotal for subsequently growing the hierarchical $\mathrm{ZnO}$ nanowire-nanosheet architectures by the ACG method. Our previous work ${ }^{26}$ demonstrated that $\mathrm{ZnO}$ crystals could nucleate and grow directly on the ITO substrate by ACG. But, herein, the potential barrier to crystal nucleation and subsequent growth for $\mathrm{ZnO}$ crystals on the ITO substrate is much higher than that on the obtained $\mathrm{ZnO}$ nanosheet arrays. Therefore, during the secondary growth, $\mathrm{ZnO}$ nanowires grew preferentially on the surface of the primary $\mathrm{ZnO}$ nanosheets rather than the ITO substrate, leading to the hierarchical $\mathrm{ZnO}$ nanowire-nanosheet architectures. However, if the as-electrodeposited $\mathrm{Zn}_{5}(\mathrm{OH})_{8} \mathrm{Cl}_{2}$ nanosheets were used as the substrate, $\mathrm{ZnO}$ nanowires would not nucleate and grow on the surface of the $\mathrm{Zn}_{5}(\mathrm{OH})_{8} \mathrm{Cl}_{2}$ nanosheets. Alternatively, $\mathrm{ZnO}$ nanorods with larger diameter directly nucleated and grew on the ITO substrate, as shown in Figure 5 , which indicates that the potential barrier to crystal nucleation and subsequent growth for $\mathrm{ZnO}$ crystals on the ITO substrate is much lower than that on the $\mathrm{Zn}_{5}(\mathrm{OH})_{8} \mathrm{Cl}_{2}$ nanosheet arrays. By comparison of Figure 3a with Figure 5, one can found that

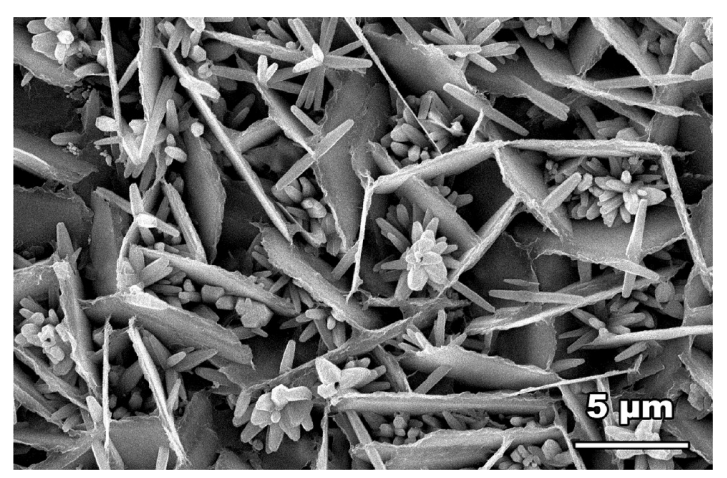

Figure 5. SEM image of the sample grown by ACG on the as-electrodeposited $\mathrm{Zn}_{5}(\mathrm{OH})_{8} \mathrm{Cl}_{2}$ nanosheet array without heat treatment.

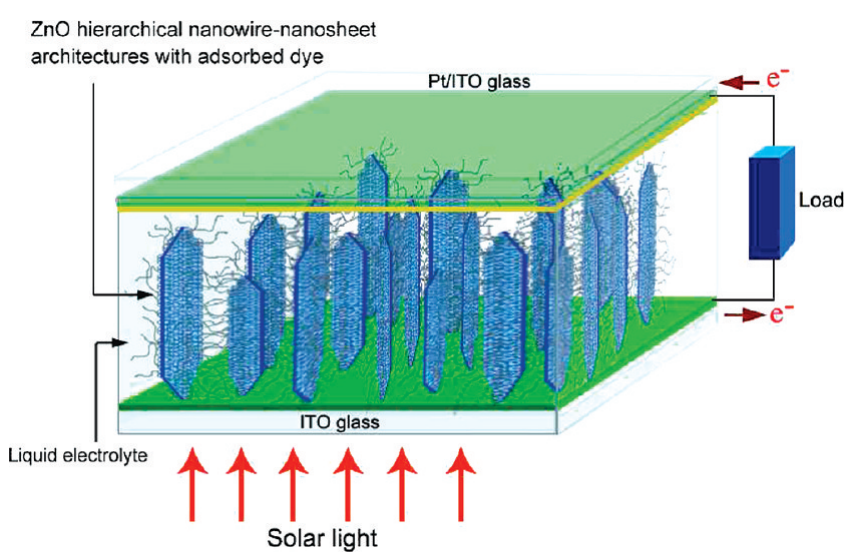

Figure 6. Schematic diagram of the DSSCs based on the hierarchical $\mathrm{ZnO}$ nanowire- nanosheet architectures.

the hierarchical nanowire-nanosheet architectures corresponding to Figure 3a obviously possess larger internal surface area.

The DSSC was assembled from the hierarchical $\mathrm{ZnO}$ nanowire-nanosheet architectures corresponding to Figure 3 obtained by ACG after a $12 \mathrm{~h}$ reaction time. The configuration of the DSSC based on the hierarchical $\mathrm{ZnO}$ nanoarchitectures resembles the schematic diagram illustrated in Figure 6. At the same time, the control experiment was also performed using the DSSC constructed by the original $\mathrm{ZnO}$ nanosheet arrays. For more impartial comparison, the two types of $\mathrm{ZnO}$ nanostructured film with nearly same thickness $(\sim 5 \mu \mathrm{m})$ were selected to prepare the DSSCs, as shown in Figure 7. Figure 8 shows the compared photocurrent-voltage $(J-V)$ characteristics of both the DSSCs under a simulated illumination with a light intensity of $100 \mathrm{~mW} / \mathrm{cm}^{2}$ (AM 1.5). The short-circuit current density $\left(J_{\mathrm{sc}}\right)$, the open-circuit voltage $\left(V_{\mathrm{oc}}\right)$, the fill factor $(F F)$, the maximum current output $\left(J_{\max }\right)$, the maximum voltage output $\left(V_{\max }\right)$, and the overall light conversion efficiency $(\eta)$ derived from the $J-V$ curves for both $\mathrm{ZnO}$ nanosheet arrays and hierarchical nanowire nanosheet-based DSSCs are summarized in Table 1. From Figure 8 and Table 1, it can be seen that the $J_{\mathrm{sc}}, V_{\mathrm{oc}}$, and $F F$ for the DSSC constructed using the hierarchical $\mathrm{ZnO}$ nanowire nanosheet architectures $\left(J_{\mathrm{sc}}=10.9 \mathrm{~mA} / \mathrm{cm}^{2}, V_{\mathrm{oc}}\right.$ $=0.68 \mathrm{~V}, F F=65 \%$ ) show obvious improvement over the DSSC constructed using the $\mathrm{ZnO}$ nanosheet arrays $\left(J_{\mathrm{sc}}=7.2\right.$ $\left.\mathrm{mA} / \mathrm{cm}^{2}, V_{\mathrm{oc}}=0.61 \mathrm{~V}, F F=52 \%\right)$. A higher $J_{\mathrm{sc}}$ would result in a higher $\eta$ since the $\eta$ is partly dependent on the $J_{\mathrm{sc}}{ }^{30}$ Therefore, because of the much improved $J_{\mathrm{sc}}$ and FF, the hierarchical nanowire-nanosheet based DSSC reached a total power conversion efficiency of $4.8 \%$, which is nearly twice as high as that of the nanosheet arrays based DSSC (2.3\%). 

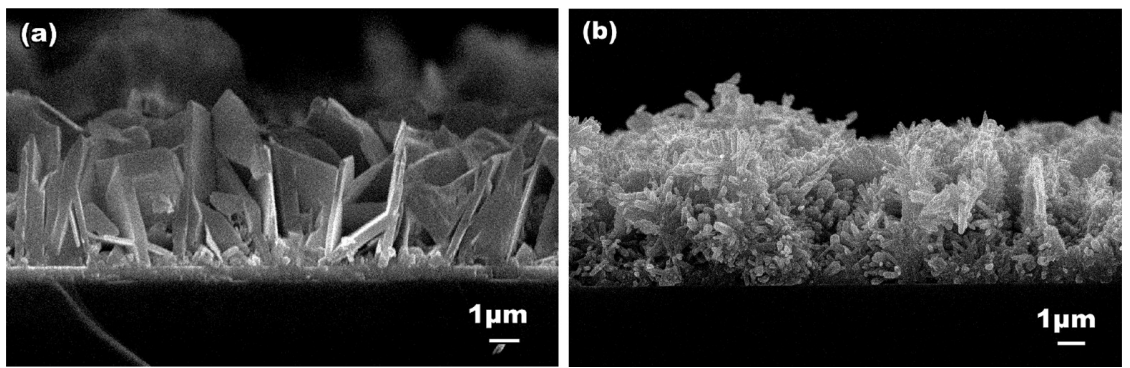

Figure 7. Cross-sectional SEM images of the two $\mathrm{ZnO}$ nanostructured film with nearly same thickness: (a) the $\mathrm{ZnO}$ nanosheet arrays, (b) the hierarchical $\mathrm{ZnO}$ nanowire-nanosheet architectures derived from the $\mathrm{ZnO}$ nanosheet arrays.

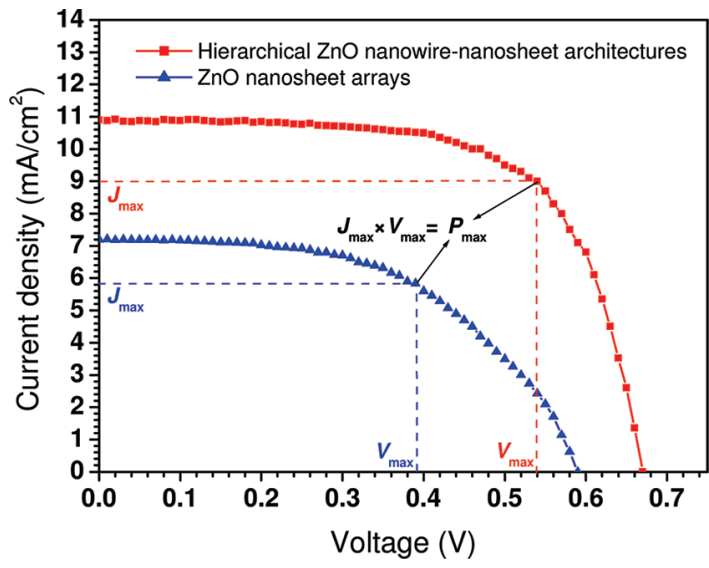

Figure 8. Current-voltage characteristics of the DSSCs constructed using the $\mathrm{ZnO}$ nanosheet arrays and the hierarchical $\mathrm{ZnO}$ nanowirenanosheet architectures derived from the $\mathrm{ZnO}$ nanosheet arrays under a simulated illumination with a light intensity of $100 \mathrm{~mW} / \mathrm{cm}^{2}$ (AM $1.5)$.

Generally, the photocurrent density in DSSCs is dependent on the initial number of photogenerated carriers, the electron injection efficiency from dye molecules to semiconductor, and the recombination rate between the injected electrons and oxidized dye or tri-iodine ions in the electrolyte..$^{31}$ The electron injection efficiency describes the probability of photogenerated electrons transferring from the dye molecules to semiconductor, which lies on the relative energy levels of the dye and semiconductor, the residential lifetime of photogenerated electrons in the dye molecules, and the density of electron-accepting states in the semiconductor. ${ }^{32}$ Thus, as far as $\mathrm{ZnO}$ sensitized with Ru-complex acidic dyes is concerned, it is thought that the electron injection efficiency is not distinctly discriminating for the given $\mathrm{ZnO}$-based DSSC systems. On the basis of the assumption of the same injection efficiency, it is reasonable that the initial number of photogenerated carriers and the recombination rate between the injected electrons and oxidized dye or tri-iodine ions, which are subjected to the influence of differently nanostructured photoanodes, may be significant factors affecting the photocurrent density. Compared to the $\mathrm{ZnO}$ nanosheets based DSSC, the increase in short-circuit current density for the hierarchical $\mathrm{ZnO}$ nanowire-nanosheet based DSSC is the enhanced light harvesting behavior associated with the enlargement of internal surface area within the photoanode, resulting in sufficient dye loading. Although the density and thickness

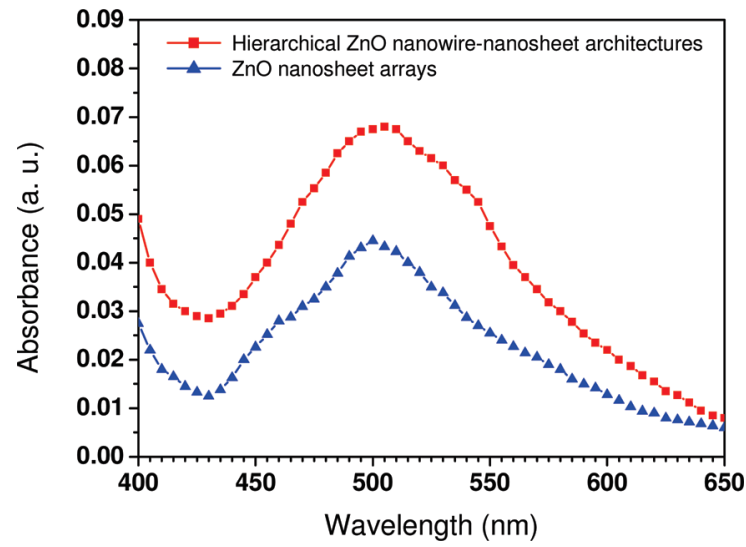

Figure 9. Optical absorbance of solutions containing dyes detached from the primary $\mathrm{ZnO}$ nanosheet array and the hierarchical $\mathrm{ZnO}$ nanowire-nanosheet architecture films (both with $0.64 \mathrm{~cm}^{2}$ area) with $0.1 \mathrm{mM} \mathrm{NaOH}$

of our original $\mathrm{ZnO}$ nanosheet arrays indirectly obtained by electrodeposition exhibit the disadvantage compared with previous report, ${ }^{21}$ herein, the hierarchical $\mathrm{ZnO}$ nanowire-nanosheet architectures derived from the primary nanosheet arrays have nanoscale wirelike branches that stretch to fill the gaps among primary $\mathrm{ZnO}$ nanosheet arrays (see Figure 3), therefore compensating the above-mentioned disadvantages and resulting in a better dye loading capability, which can be verified by subsequent desorption experiment. Figure 9 shows the optical absorbance of solutions containing dyes detached from the primary $\mathrm{ZnO}$ nanosheet arrays and the hierarchical $\mathrm{ZnO}$ nanowire-nanosheet architecture films (both with $0.64 \mathrm{~cm}^{2}$ area) in a $10-\mathrm{mL}$ quartz cell with $0.1 \mathrm{mM} \mathrm{NaOH}$. The amount of dye loading in the hierarchical $\mathrm{ZnO}$ nanowire-nanosheet architecture was found to be $4.7 \times 10^{-9} \mathrm{~mol} / \mathrm{cm}^{2}$, which is slightly higher than the value of $3.9 \times 10^{-9} \mathrm{~mol} / \mathrm{cm}^{2}$ corresponding to the photoanode with the dyed $\mathrm{ZnO}$ nanosheet arrays, even though the overall light conversion efficiency of the DSSC constructed using the hierarchical $\mathrm{ZnO}$ nanowire-nanosheet architectures is twice as high as that of the DSSC constructed using the bare $\mathrm{ZnO}$ nanosheet arrays. This phenomenon was previously encountered in $\mathrm{ZnO}$ nanowire-based DSSCs by Cheng et al. ${ }^{18}$ and Jiang et al. ${ }^{19}$ Similarly, we also regard this phenomenon (small difference in dye loading amount but great difference in $\eta$ for both the DSSCs) as the formation of $\mathrm{Zn}^{2+}$ / dye complex in the gaps due to the insufficient internal surface

TABLE 1: Photovoltaic Parameters of the DSSCs on the Basis of ZnO Films Consisting of Hierarchical Nanowire-Nanosheet Architectures and Nanosheet Arrays, Respectively

\begin{tabular}{|c|c|c|c|c|c|c|}
\hline $\mathrm{ZnO}$ film & $V_{\mathrm{oc}}(\mathrm{V})$ & $J_{\mathrm{sc}}\left(\mathrm{mA} / \mathrm{cm}^{2}\right)$ & $V_{\max }(\mathrm{V})$ & $J_{\max }\left(\mathrm{mA} / \mathrm{cm}^{2}\right)$ & $\mathrm{FF}(\%)$ & $\eta(\%)$ \\
\hline hierarchical nanowire-nanosheet architectures & 0.68 & 10.9 & 0.54 & 9.0 & 65 & 4.8 \\
\hline nanosheet arrays & 0.61 & 7.2 & 0.39 & 5.8 & 52 & 2.3 \\
\hline
\end{tabular}


of $\mathrm{ZnO}$ nanosheet arrays and the excess dye during dye loading process, instead of the effective chemical bonding between $\mathrm{ZnO}$ and dye molecules. ${ }^{33}$ The $\mathrm{Zn}^{2+}$ /dye complex can absorb the light but not efficiently inject photogenerated electrons into the $\mathrm{ZnO}$ electrode. In fact, the color of the dyed $\mathrm{ZnO}$ nanosheet arrays became lighter if rinsed by ethanol after they have been rested. When they are reused as the DSSC photoanode and retested, a similar photocurrent density could be obtained, indicating that not all of the original dye molecules adsorbed on the $\mathrm{ZnO}$ nanosheets could effectively inject photogenerated electrons into the $\mathrm{ZnO}$ nanosheets. The $\mathrm{Zn}^{2+} /$ dye complex could agglomerate to form a thick covering layer instead of a monolayer, and is thus inactive for electron injection.

Moreover, the recombination rate between the injected electrons and oxidized dye or tri-iodine ions in the electrolyte is another important factor affecting the photocurrent density and the performance of the DSSCs. Hosono et al. ${ }^{21}$ thought that similar to $1 \mathrm{D} \mathrm{ZnO}$ nanostructures, vertically aligned $2 \mathrm{D} \mathrm{ZnO}$ nanosheet arrays could also provide a direct conduction pathway for the rapid collection of photogenerated electrons. In addition, the gaps between the upright-standing $\mathrm{ZnO}$ nanosheets span from the substrate to the top of each sheet. Hence, the facile transport of the redox couple $\left(\mathrm{I}^{-} / \mathrm{I}_{3}{ }^{-}\right)$between the electrodes through the vertical gaps decreases the resistance of the cell and the recombination of the injected electrons. However, according to the suggestion by Jiang et al., ${ }^{19}$ upstanding arrays (including $1 \mathrm{D}$ or 2D $\mathrm{ZnO}$ nanostructures) may not be favorable for light-harvesting or light-dye interactions because some photons could possibly fall on the gaps between adjacent $\mathrm{ZnO}$ nanosheets (as shown in Figure 10a), not being able to be absorbed by the dye interfacing $\mathrm{ZnO}$ nanosheets. Therefore, the optical loss may be significantly great for upstanding arrays. Herein, our hierarchical $\mathrm{ZnO}$ nanowire-nanosheet architectures derived from $\mathrm{ZnO}$ nanosheet arrays simultaneously give attentions to the problems encountered by them. First, the hierarchical $\mathrm{ZnO}$ nanowire-nanosheet architectures still retain the vertical sheetlike skeletons, as shown in Figure 3a. The photogenerated electrons from the dye adsorbed on the randomly oriented $\mathrm{ZnO}$ nanowires can converge on the sheetlike skeletons and then transfer to the collection electrode along the vertical sheetlike skeletons. The possible electron transport mechanism in the DSSC photoanode consisting of the hierarchical $\mathrm{ZnO}$ nanowirenanosheet architectures is schematically illustrated in Figure 11. It can be seen from Figure 11 that the random wirelike branches

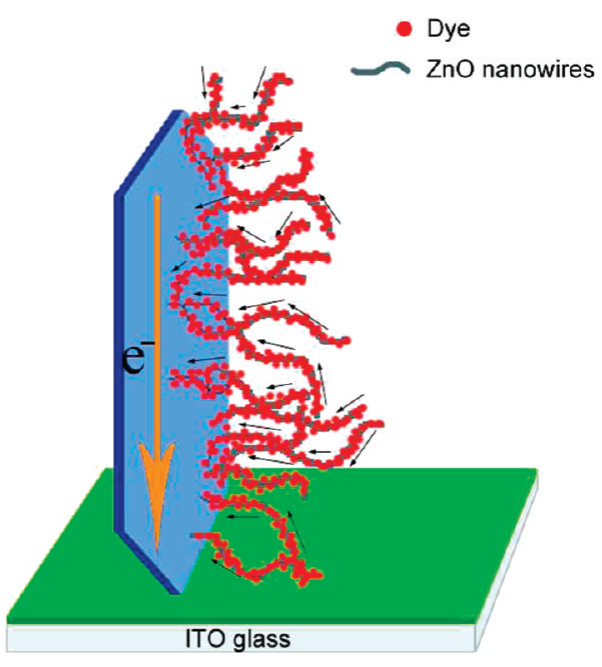

Figure 11. Schematic diagram of the possible electron transport mechanism in the DSSC photoanode consisting of the hierarchical $\mathrm{ZnO}$ nanowire-nanosheet architectures.

are advantageous for a larger internal surface area and increased light-dye interactions, meanwhile, not sacrificing the good electron transportation similar to upstanding nanosheet arrays and ensuring the low recombination rate between the injected electrons and oxidized dye due to relatively rapid collection of photogenerated electrons. Second, although the gaps between the upright $\mathrm{ZnO}$ nanosheets have been filled with densely and randomly distributed $\mathrm{ZnO}$ nanowires, large numbers of interstice still exist (see Figure $3 b$ ) and can provide the space enough to transport the redox couple $\left(\mathrm{I}^{-} / \mathrm{I}_{3}{ }^{-}\right)$between the electrodes. Likewise, this can also reduce the recombination rate between the injected electrons and tri-iodine ions in the electrolyte. Third, the hierarchical $\mathrm{ZnO}$ nanowire-nanosheet architectures with dye loading can make the best of the photons falling on the gap between adjacent $\mathrm{ZnO}$ nanosheets (as shown in Figure 10b), avoiding the problem presented by Jiang et al. ${ }^{19}$

In previous reports, the low $F F$ was ascribed to the recombination of charges at the interface between $\mathrm{ZnO}$ nanostructures and $\mathrm{I}^{-} / \mathrm{I}_{3}{ }^{-}$electrolyte, ${ }^{19}$ and the photoshunt. ${ }^{7}$ However, as shown in Table 1, the hierarchical $\mathrm{ZnO}$ nanowire-nanosheet based DSSC show a much better $F F(65 \%)$ in comparison with that of the nanosheet arrays based one. As to the recombination of charges, we have discussed in detail. In the present DSSC based
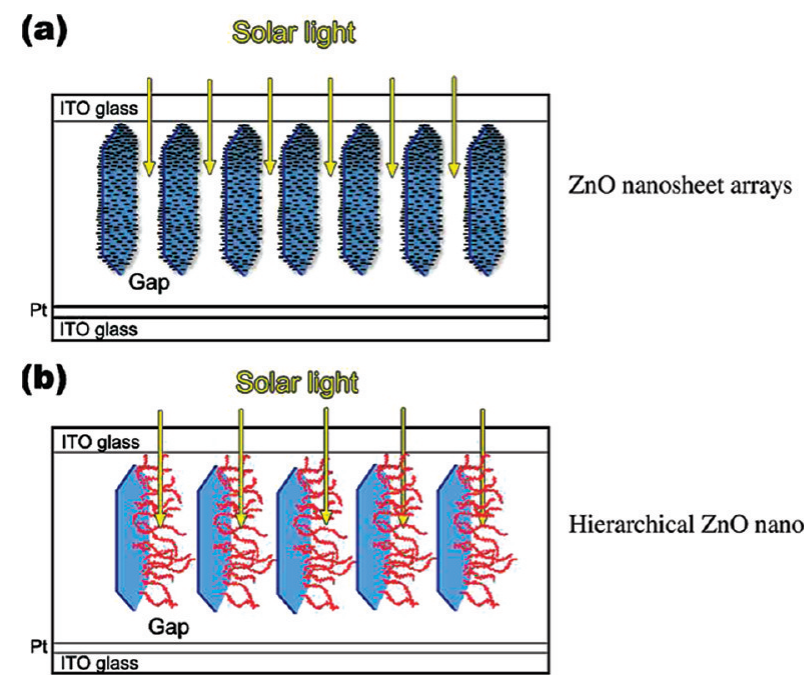

Hierarchical $\mathrm{ZnO}$ nanowire-nanosheet architectures

Figure 10. Schematic diagram of (a) the upstanding $\mathrm{ZnO}$ nanosheet arrays and (b) the hierarchical $\mathrm{ZnO}$ nanowire-nanosheet architectures based DSSCs shined under solar light. 
on the hierarchical $\mathrm{ZnO}$ nanowire-nanosheet architectures, a low recombination rate between the injected electrons and oxidized dye or tri-iodine ions in the electrolyte was obtained. Besides, our preparation method could also result in the high $F F$ due to the improvement in the shunt resistance. Baxter et al. ${ }^{7}$ compared the shunt resistance of solar cells constructed using the nanowire grown by hydrothermal method with that of solar cells made from the nanowire grown by metalorganic chemical vapor deposition (MOCVD), indicating that the photoshunt may be at the nanowire-F: $\mathrm{SnO}_{2}$ interface. Shunt resistance of the MOCVD grown nanowires are better owing to a thick polycrystalline $\mathrm{ZnO}$ layer that grows on the $\mathrm{F}: \mathrm{SnO}_{2}$ prior to nucleation and growth of the nanowires. The thick polycrystalline $\mathrm{ZnO}$ layer was dense and could keep the electrolyte from penetrating to the $\mathrm{F}: \mathrm{SnO}_{2}$. According to their explanation, Similarly, our electrodeposition could also result in a thick polycrystalline $\mathrm{Zn}_{5}(\mathrm{OH})_{8} \mathrm{Cl}_{2}$ layer prior to $\mathrm{Zn}_{5}(\mathrm{OH})_{8} \mathrm{Cl}_{2}$ nanosheet growth. The polycrystalline $\mathrm{Zn}_{5}(\mathrm{OH})_{8} \mathrm{Cl}_{2}$ layer was transformed into dense $\mathrm{ZnO}$ layer during the thermal treatment at $350{ }^{\circ} \mathrm{C}$ or above in air, also keeping the electrolyte from penetrating to the $\mathrm{In}: \mathrm{SnO}_{2}$. Therefore, the hierarchical nanowire-nanosheet architectures and the preparation method used should be responsible for the obtained high $F F$.

The hierarchical nanowire-nanosheet architectures could not only help in the better dye-loading but also help in increasing the optical path length within the photoanode due to multiple scattering. In general, highly disordered structures result in multiple scattering of the light traveling, possibly leading to photon localization because of the formation of traps for optical confinement. Herein, the disordered $\mathrm{ZnO}$ nanowires grown randomly on $\mathrm{ZnO}$ nanosheets are in accordance with the structure characteristic. A similar opinion was presented by Zhang et al. in their recent researches on aggregation of $\mathrm{ZnO}$ nanocrystals..$^{30,34,35}$ They have attributed the improvement in $\eta$ to the increase in the absorption coefficient owing to the increased scattering effect in the $\mathrm{ZnO}$ aggregated compared to nonaggregated $\mathrm{ZnO}$ films. However, in the case of our hierarchical nanowire-nanosheet architectures, further study is still needed and under way.

\section{Conclusions}

In conclusion, hierarchical $\mathrm{ZnO}$ nanowire-nanosheet architectures for use in highly efficient DSSCs have been achieved. We have demonstrated that the DSSC based on hierarchical ZnO nanowire-nanosheet architectures with better dye loading and light harvesting showed a power conversion efficiency of $4.8 \%$, which is nearly twice as high as that of the DSSCs constructed using the primary $\mathrm{ZnO}$ nanosheet arrays. The improvement in the photovoltaic performance can benefit from the enlargement of the internal surface area within the photoanode without sacrificing a direct conduction pathway for the rapid collection of photogenerated electrons. Further, the concept of the hierarchical nanowire-nanosheet architectures is anticipated to be applicable to other semiconductor photoelectrodes in organicinorganic nanocomposite solar cells and hierarchical heterojunction nanostructures for future potential applications.
Acknowledgment. This work was supported by the National Basic Research Programme of China (No. 2006CB300404) and the National Natural Science Foundation of China (No. 60976003). Jiangsu Planned Projects for Postdoctoral Research Funds (No. 0902003B).

\section{References and Notes}

(1) Huang, L. S.; Wright, S.; Yang, S. G.; Shen, D. Z.; Gu, B. X.; Du, Y. W. J. Phys. Chem. B 2004, 108, 19901.

(2) Khan, A.; Jadwisienczak, W. M.; Kordesch, M. Physica E 2006, 33,331 . 1047.

(3) Lu, F.; Cai, W. P.; Zhang, Y. G. Adv. Funct. Mater. 2008, 18,

(4) Ye, Z. Z.; Lu, J. G.; Zhang, Y. Z.; Zeng, Y. J.; Chen, L. L.; Zhuge, F.; Yuan, G. D.; He, H. P.; Zhu, L. P.; Huang, J. Y.; Zhao, B. H. Appl. Phys. Lett. 2007, 91, 113503.

(5) Wang, Z. L.; Song, J. H. Science 2006, 312, 242.

(6) Law, M.; Greene, L. E.; Johnson, J. C.; Saykally, R.; Yang, P. D. Nat. Mater. 2005, 4, 455

(7) Baxter, J. B.; Walker, A. M.; Ommering, K. V.; Aydil, E. S. Nanotechnology 2006, 17, S304.

(8) Zhu, L.; Fan, Y. Q.; Zhao, M. C.; Wu, M.; Zhang, J. Y.; Xu, C. X.; Cui, Y. P. Chin. Phys. Lett. 2009, 26, 018401.

(9) Hagfeldt, A.; Gratzel, M. Acc. Chem. Res. 2000, 33, 269.

(10) Gratzel, M. Nature 2001, 414, 338.

(11) Quintana, M.; Edvinsson, T.; Hagfeldt, A.; Boschloo, G. J. Phys. Chem. C 2007, 111, 1035 .

(12) Keis, K.; Bauer, C.; Boschloo, G.; Hagfeldt, A.; Westermark, K.; Rensmo, H.; Siegbahn, H. J. Photochem. Photobiol., A 2002, 148, 57.

(13) Keis, K.; Magnusson, E.; Kindstrom, H.; Lindquist, S. E.; Hagfeldt, A. Sol. Energ. Mater. Sol. Cell. 2002, 73, 51.

(14) Greene, L. E.; Yuhas, B. D.; Law, M.; Zitoun, D.; Yang, P. D. Inorg. Chem. 2006, 45, 7535.

(15) Pradhan, B.; Batabyal, S. K.; Pal, A. J. Sol. Energ. Mater. Sol. Cell. 2007, 91, 769.

(16) Cheng, A. J.; Tzeng, Y. H.; Zhou, Y.; Park, M.; Wu, T. H.; Shannon, C.; Wang, D.; Lee, W. W. Appl. Phys. Lett. 2008, 92, 092113. (17) Martinson, A. B. F.; Elam, J. W.; Hupp, J. T.; Pellin, M. J. Nano Lett. 2007, 7, 2183.

(18) Cheng, H. M.; Chiu, W. H.; Lee, C. H.; Tsai, S. Y.; Hsieh, W. F. J. Phys. Chem. C 2008, 112, 16359.

(19) Jiang, C. Y.; Sun, X. W.; Lo, G. Q.; Kwong, D. L. Appl. Phys. Lett. 2007, 90, 263501.

(20) Ku, C. H.; Wu, J. J. Nanotechnology 2007, 18, 505706.

(21) Hosono, E.; Fujihara, S.; Honma, I.; Zhou, H. S. Adv. Mater. 2005, 17,2091 .

(22) Lin, C. F.; Lin, H.; Li, J. B.; Li, X. J. Alloys Compd. 2008, 462, 175.

(23) Hsu, Y. F.; Xi, Y. Y.; Yip, C. T.; Djurisic, A. B.; Chan, W. K. J. Appl. Phys. 2008, 103, 083114.

(24) Chen, W.; Zhang, H. F.; Hsing, I. M.; Yang, S. H. Electrochem. Commun. 2009, 11, 1057.

(25) Xu, F.; Lu, Y. N.; Xie, Y.; Liu, Y. F. Mater. Des. 2009, 30, 1704.

(26) Xu, F.; Lu, Y. N.; Xie, Y.; Liu, Y. F. J. Phys. Chem. C 2009, 113 , 1052 .

(27) Das, J.; Evans, I. R.; Khushalani, D. Inorg. Chem. 2009, 48, 3508.

(28) Zhang, J. W.; Zhu, P. L.; Li, J. H.; Chen, J. M.; Wu, Z. S.; Zhang, Z. J. Cryst. Growth Des. 2009, 9, 2329.

(29) Huang, Q. L.; Wang, M.; Zhong, H. X.; Chen, X. T.; Xue, Z. L.; You, X. Z. Cryst. Growth Des. 2008, 8, 1412.

(30) Chou, T. P.; Zhang, Q. F.; Fryxell, G. E.; Cao, G. Z. Adv. Mater. 2007, 19, 2588.

(31) Rao, R. A.; Dutta, V. Nanotechnology 2008, 19, 445712.

(32) Zhang, Q. F.; Dandeneau, C. S.; Zhou, X. Y.; Cao, G. Z. Adv. Mater. 2009DOI: 10.1002/adma.200803827.

(33) Horiuchi, H.; Katoh, R.; Hara, K.; Yanagida, M.; Murata, S.; Arakawa, H.; Tachiya, M. J. Phys. Chem. B 2003, 107, 2570.

(34) Zhang, Q. F.; Chou, T. P.; Russo, B.; Jenekhe, S. A.; Cao, G. Z. Adv. Funct. Mater. 2008, $18,1654$.

(35) Zhang, Q. F.; Chou, T. P.; Russo, B.; Jenekhe, S. A.; Cao, G. Z. Angew. Chem., Int. Ed. 2008, 47, 2402.

JP910363W 